Check for updates

Cite this: Analyst, 2019, 144, 212

\title{
Poly(amidoamine)-coated magnetic particles for enhanced detection of Schistosoma circulating anodic antigen in endemic urine samples
}

\author{
Christine F. Markwalter, ${ }^{\text {a }}$ Paul L. A. M. Corstjens, ${ }^{b}$ Claire M. Mammoser, ${ }^{\text {a }}$ \\ Garrett Camps, ${ }^{a}$ Govert J. van Dam ${ }^{*} \dagger^{\mathrm{C}}$ and David W. Wright (D) $\star^{\mathrm{a}}$
}

\begin{abstract}
Accurate and sensitive point-of-care diagnostic tools are critical for schistosomiasis control and elimination. The existing ultrasensitive lateral flow assay for the detection of Schistosoma circulating anodic antigen (CAA) has demonstrated excellent sensitivity but is time-consuming and requires significant laboratory infrastructure that limits its applicability at the point of care. To address this challenge, we sought to develop an alternative sample preparation method to concentrate CAA from large-volume urine samples requiring little-to-no laboratory equipment. The developed method relies on electrostatic interactions between the negatively-charged CAA biomarker and positively-charged poly(amidoamine) (PAMAM) dendrimers functionalized to the surface of magnetic particles. After CAA capture on the surface of the PAMAM-functionalized magnetic beads, the supernatant was removed, and CAA was eluted into a small-volume, high-salt elution buffer. This concentrated eluate was subsequently applied to the existing lateral flow assay. The PAMAM-functionalized magnetic bead-based CAA concentration method was extensively characterized for its robustness, evaluated on a set of endemic urine samples, and compared to spin filter-based concentration methods. The novel bead-based sample preparation method used only disposable laboratory materials, resulted in a 200 -fold improvement in CAA limits of detection, and performed just as well as infrastructure-intensive and high-cost spin filter methods. Additionally, the functionalized beads were robust to variations in sample $\mathrm{pH}$ and storage conditions. The PAMAM-functionalized magnetic bead-based CAA concentration method represents a promising step toward ultrasensitive schistosomiasis diagnosis at the point of care.
\end{abstract}

Received 21st May 2018, Accepted 24th July 2018 DOI: 10.1039/c8an00941d rsc.li/analyst somiasis in select regions and countries by $2025 .^{3}$ Accomplishing these goals, particularly transmission interruption, will rely heavily on the development of accurate and fielddeployable schistosomiasis diagnostics. Precise mapping of Schistosoma prevalence will be necessary to identify foci for targeted interventions. Strong surveillance will be needed to evaluate the success of these interventions, and as prevalence decreases, detecting low-burden asymptomatic cases will become increasingly important. ${ }^{4}$

Egg microscopy is the most commonly employed tool for schistosomiasis diagnosis. The Kato-Katz technique is used most often to prepare stool samples for inspection for schistosome eggs, and urine filtration methods are used to concentrate eggs from large urine volumes before inspection by microscopy. Both of these methods can be cumbersome and labor-intensive and lack the sensitivity to detect low-burden infections, which can contribute to ongoing transmission. ${ }^{5}$ Additionally, egg counts are highly susceptible to day-to-day variation in egg output and can be unreliable even for identifying high-intensity infections. ${ }^{6}$ 
Paper-based lateral flow assays (LFAs) are attractive alternatives to microscopy because they are easy-to-use, low-cost, and disposable. Two antigens generated by schistosomes have proven useful for urine and blood-based detection by LFA: the circulating cathodic antigen (CCA) and the circulating anodic antigen (CAA). These heavily glycosylated proteoglycans are named for their overall positive and negative charges, respectively, at physiological pH. A urine-based CCA-specific LFA (POC-CCA) developed by van Dam et al. is now commercially available and has been extensively evaluated in the field, demonstrating good sensitivity for the detection of S. mansoni infections compared to Kato-Katz. ${ }^{7-16}$ In this format, a urine sample is deposited onto a conjugate pad, where targetspecific antibodies conjugated to gold nanoparticles bind to CCA in the sample. Running buffer then carries these complexes down a nitrocellulose membrane, and they reach a line of immobilized capture antibodies, resulting in a visible line of gold nanoparticles only when CCA is present. Although the POC-CCA is a useful alternative to microscopy for monitoring and evaluating $S$. mansoni control, the sensitivity of the test remains too low to be applied in elimination campaigns in low-transmission settings. ${ }^{8}$

Recently, a more sensitive schistosomiasis LFA has been developed for the detection of CAA, which is present in the serum and urine of patients with Schistosoma infections of all known species and can be used as a direct measure of worm burden. ${ }^{17-19}$ This assay is formatted similarly to a conventional lateral flow assay, with the exception that up-converting nanoparticles (UCPs) are used as signaling elements rather than gold nanoparticles. These $400 \mathrm{~nm} \mathrm{Y}_{2} \mathrm{O}_{2} \mathrm{~S}_{:} \mathrm{Yb}^{3+}, \mathrm{Er}^{3+}$ nanoparticles, which are excited with infrared light $(980 \mathrm{~nm})$ and emit green light $(550 \mathrm{~nm})$, are advantageous because this high anti-stokes shift does not occur in nature. ${ }^{20}$ Thus, background autofluorescence typically associated with biological samples and assay components is effectively eliminated. The UCPbased LFA for CAA (UCF-LF CAA) was shown to be more sensitive than a laboratory-based enzyme-linked immunosorbent assay (ELISA) for CAA. ${ }^{17}$ To further increase the analytical sensitivity of the test, Corstjens et al. added a pre-concentration step to the sample preparation method. ${ }^{21}$ Using centrifugal spin-filters, CAA in urine samples as large as $7.5 \mathrm{ml}$ was concentrated to just $20 \mu \mathrm{l}$ before running the UCP-LF CAA assay. This resulted in an improvement in the limit of detection of up to 333 -fold; the test was capable of detecting $0.03 \mathrm{pg} \mathrm{ml}^{-1}$ CAA in urine, 100 times lower than serum CAA levels predicted for a single worm pair. ${ }^{21}$ This ultrasensitive method has been successfully applied to $2 \mathrm{ml}$ endemic urine samples from highand low-transmission areas, indicating prevalences much higher than those measured by microscopy. ${ }^{21-24}$ Additionally, the ultrasensitive UCP-LF CAA assay has demonstrated promise for sample pooling strategies that would allow for large-scale testing, enabling precision mapping for targeted interventions while decreasing overall assay cost. ${ }^{25}$

In the shift from morbidity control to elimination, detection of low-burden Schistosoma infections at the point of care will become increasingly important. As such, there is a press- ing need for highly sensitive schistosomiasis detection tools to be applicable in low-resource settings. The ultrasensitive UCP-LF CAA test requires laboratory infrastructure to carry out the assay, particularly sample concentration. Centrifugation steps are needed in order to perform the TCA extraction and concentrate large-volumes of urine. For samples $2 \mathrm{ml}$ and greater, temperature-controlled centrifugation is needed to prevent overheating due to extended centrifugation times. This restricts the utility of the ultrasensitive UCP-LF CAA assay in its current format to well-equipped reference laboratories or tertiary facilities. Thus, the utility of the ultrasensitive assay at the point of care depends on the development of alternative methods for concentration of CAA from urine samples.

To address this need, we developed an alternative largevolume urine sample preparation method for the UCP-LF CAA assay that requires little-to-no laboratory equipment. The new method relies on electrostatic interactions between magnetic particles functionalized with positively-charged poly(amidoamine) (PAMAM) dendrimers and highly negatively-charged CAA in urine. Once CAA was captured on the surface of the PAMAM-functionalized magnetic beads, which could be manipulated with an external magnet, the supernatant was removed, and CAA concentrated into a small volume with a high-salt elution buffer. This concentrated, CAA-containing eluate was subsequently applied to the UCP-LF CAA assay. The PAMAM bead-based sample concentration method achieved full theoretical enhancement compared to the unenhanced UCP-LF CAA protocol. Additionally, the method was successfully applied to a panel of 15 patient samples and demonstrated equal performance compared to the laboratory-based spin column method.

\section{Materials and methods}

\section{Materials and reagents}

Dynabeads ${ }^{\circledR}$ MyOne ${ }^{\mathrm{TM}}$ Carboxylic Acid magnetic particles were purchased from ThermoFisher Scientific (65012). All poly (amidoamine) dendrimers were purchased from SigmaAldrich. Proprietary mouse monoclonal anti-CAA antibodies ( $\alpha$-CAA; clone 147-3G4-A) were available from the Department of Parasitology, Leiden University Medical Center. ${ }^{26}$ Horseradish peroxidase (HRPx) was conjugated to $\alpha$-CAA $\left(\alpha\right.$-CAA:HRPx) using EZ-Link ${ }^{\mathrm{TM}}$ Plus Activated Peroxidase Kit (ThermoFisher Scientific 31489). CAA standards were derived from the TCA-soluble fraction of Schistosoma Adult Worm Antigen (AWA-TCA), which contains approximately 3\% w/w CAA. $^{27}$ TMB One was purchased from Promega (G7431). A Cole-Palmer vortex mixer (UX-04726-01) with a modified ThermoFisher $15 \mathrm{~mm}$ tube-holder (88880122) was used for all vortexed incubations. Absorbance was measured on a Biotek Synergy $\mathrm{H} 4$ microplate reader (Vanderbilt University) or a MultiSkan $^{\mathrm{TM}}$ FC Microplate Photometer (Leiden University Medical Center). All other reagents and materials were purchased from either Fisher Scientific or Sigma Aldrich. 


\section{Bead preparation}

Dynabeads ${ }^{\circledR}$ MyOne ${ }^{\mathrm{TM}}$ Carboxylic Acid magnetic particles $\left(1 \mathrm{ml}, 10 \mathrm{mg} \mathrm{ml}^{-1}\right)$ were washed three times with $0.1 \mathrm{M} 2-(N$ morpholino)ethanesulfonic acid (MES) pH 5. On the final wash, the supernatant was removed, and a $1 \mathrm{ml}$ solution of $78 \mathrm{mM}$ NHS, $31 \mathrm{mM}$ EDC, and $78 \mu \mathrm{M}$ PAMAM generation 4.0 in 0.1 M MES pH 5 was added to the beads, which were then vortexed and incubated on a rotisserie for 2 hours at room temperature. Next, the beads were washed 3 times with $1 \mathrm{ml}$ of MES pH 5 before a 15-minute incubation with $10 \mathrm{mM}$ hydroxyl amine in MES pH 5. Finally, the beads were washed 3 times and re-suspended in MES pH 5 with $0.1 \%$ Tween-20.

\section{CAA enzyme-linked immunosorbent assay (ELISA)}

The ELISA protocol for CAA detection was based on previous reports. $^{28,29}$ Briefly, MaxiSorp plates (Thermo Scientific 430341) were coated with $75 \mu \mathrm{l}$ of $2 \mu \mathrm{g} \mathrm{ml} \mathrm{m}^{-1}$ mouse monoclonal $\alpha$-CAA (147-3G4-A) in $1 \times$ phosphate buffered saline (PBS). The plate was then washed with 4 times with $150 \mu \mathrm{l} 20 \times$ diluted PBS before blocking with $100 \mu \mathrm{l} 0.33 \mathrm{mg} \mathrm{ml}^{-1} \mathrm{BSA}$ in $1 \times$ PBS. After washing 4 times, $50 \mu \mathrm{l}$ samples were added to the plate. Next, the plate was washed 4 times, and incubated with $50 \mu \mathrm{l}$ of $1 \mu \mathrm{g} \mathrm{ml} \mathrm{m}^{-1} \alpha$-CAA:HRPx. After washing, $100 \mu \mathrm{l}$ of TMB One Solution was added to the plate, and the reaction was quenched with $100 \mu \mathrm{l} 2 \mathrm{M} \mathrm{H}_{2} \mathrm{SO}_{4}$ after 10 minutes. Absorbance was measured at $450 \mathrm{~nm}$. Unless noted otherwise, all reagents were diluted in PBS with $0.3 \%$ Tween-20 and $0.1 \%$ bovine serum albumin (PBST with $0.1 \%$ BSA). All incubation steps were carried out on a heated plate shaker for 15 minutes. Standard curves consisting of serial dilutions of AWA-TCA were included on each plate to enable CAA quantitation.

\section{UCP-LF CAA assay (UCAA10)}

The UCP-based lateral flow assay for CAA was performed as previously described. ${ }^{17}$ Briefly, $50 \mu \mathrm{l} 4 \% \mathrm{w} / \mathrm{v}$ trichloroacetic acid (TCA) was added to an equal volume of urine in a $1.5 \mathrm{ml}$ microcentrifuge tube, vortexed, incubated for 5 minutes, and subsequently centrifuged for $5 \mathrm{~min}$ at $13000 \mathrm{rpm}$. The mouse monoclonal (147-3G4-A) $\alpha$-CAA-functionalized $400 \mathrm{~nm}$ $\mathrm{Y}_{2} \mathrm{O}_{2} \mathrm{~S}: \mathrm{Yb}^{3+}, \mathrm{Er}^{3+}$ UCP particles were sonicated and diluted to $1 \mu \mathrm{g} \mathrm{ml}^{-1}$ in UCP-LF CAA running buffer (200 mM Tris $\mathrm{pH} 8$, $270 \mathrm{mM} \mathrm{NaCl}, 0.5 \%$ Tween-20, 1\% BSA). Next, $20 \mu \mathrm{l}$ of the clear TCA extraction supernatant was combined with $100 \mu \mathrm{l}$ of the prepared UCP particles in a 96-well plate and incubated on an orbital shaker at $37{ }^{\circ} \mathrm{C}$ for $1 \mathrm{~h}$. Lateral flow strips with mouse monoclonal (147-3G4-A) $\alpha$-CAA test lines and $\alpha$-mouse IgG control lines were added to the wells. The strips were allowed to develop and dry before scanning on a Packard FluoroCount microplate reader adapted with an IR laser (980 $\mathrm{nm})$ modified to scan lateral flow strips. Emission was measured at $550 \mathrm{~nm}$.

\section{Enhancement of UCP-LF CAA assay with PAMAM-} functionalized magnetic beads

To perform the bead-based enhancement protocol, $400 \mu \mathrm{l}$ of $12 \% \mathrm{w} / \mathrm{v}$ TCA was added to a $2 \mathrm{ml}$ urine sample in a $5 \mathrm{ml}$ microcentrifuge tube. The sample was then filtered through a $0.45 \mu \mathrm{m}$ cellulose acetate syringe filter (VWR 28145-479) and neutralized with $200 \mu \mathrm{l} 0.5 \mathrm{M} \mathrm{Na}_{3} \mathrm{PO}_{4}$. Next, $50 \mu \mathrm{l}$ of prepared PAMAM-functionalized magnetic beads $\left(10 \mathrm{mg} \mathrm{ml} \mathrm{ml}^{-1}\right)$ was added to the sample and vortexed for 5 minutes. Using a custom 3D-printed adaptor, a MagnaRack ${ }^{\mathrm{TM}}$ (Invitrogen) was used to separate magnetic particles and remove the supernatant. Next, $70 \mu \mathrm{l}$ of UCP-LF CAA running buffer was added to the beads, which were then vortexed for 5 minutes for CAA elution. The resulting supernatant was then run according to the UCP-LF CAA protocol as described above, with the exception that $50 \mu \mathrm{l}$ of $2 \mu \mathrm{g} \mathrm{ml} \mathrm{m}^{-1}$ UCP particles was combined with the $70 \mu \mathrm{l}$ magnetic bead eluate before subsequent incubation and addition of the lateral flow strips.

\section{Patient samples}

The patient urine samples analyzed in this study were collected in 1989-1990 for a previous study ${ }^{29}$ and have since been stored at $-20^{\circ} \mathrm{C}$. Informed consent was obtained from all individuals who provided samples. In total, 15 patient samples were evaluated for the present study. All patients were Egyptian males and fell within the age range of 10-49 years of age.

\section{Analysis of patient samples}

All patient samples were processed using the bead enhancement protocol described above with the exception that samples were diluted 10-fold with DI water after neutralization. Thus, PAMAM-functionalized beads were added to $2 \mathrm{ml}$ of $10 \times$ diluted samples ( $200 \mu \mathrm{l}$ of urine total). In addition to the bead enhancement protocol, all samples were processed using the standard UCAA10 protocol as well as a spin filter-based concentration protocol (UCAA200) for comparison purposes. For the latter, $200 \mu \mathrm{l}$ of $4 \%$ TCA was added to an equal volume of urine, and the supernatant resulting from the TCA extraction was placed in a $0.5 \mathrm{ml}$ spin filter with a $10 \mathrm{kDa}$ molecular weight cut-off (Amicon Ultra, Millipore Sigma UFC5010BK). After concentration (15 $\mathrm{min}$ at $14000 \mathrm{~g}$ ), $20 \mu \mathrm{l}$ of concentrate was combined with $100 \mu \mathrm{l}$ of $1 \mu \mathrm{g} \mathrm{ml}^{-1}$ UCP particles, and the UCP-LF CAA protocol was performed as usual. For all three protocols applied to patient samples, standard curves of AWA-TCA spiked in non-endemic control urine were processed simultaneously to calculate CAA concentrations. Additionally, urine reagent strips (LW Scientific URS-10) were used to determine the specific gravity of all patient samples.

\section{Data analysis}

For optimization experiments (signal measured by ELISA), CAA concentrations were interpolated from the line of best fit based on standard curves run on each plate. All standard curves run on the UCP-LF CAA assay were fit with 4-parameter logistic curves, regardless of sample preparation method employed. Limits of detection were calculated as the concentration at which the assay signal was signal $_{\text {blank }}+$ $3 \mathrm{SD}_{\text {blank }}$. All error bars represent the standard error of measurement. 


\section{Results}

\section{Optimization of PAMAM-functionalized magnetic beads}

Several parameters of the PAMAM-functionalized beads were optimized to maximize CAA capture and recovery. For all optimization experiments, the CAA ELISA was used to measure biomarker capture and recovery. Capture efficiency was determined by measuring the difference in CAA concentration between the original AWA-TCA-spiked sample and the supernatant after incubation with PAMAM-functionalized particles. Percent recovery was calculated based on the ratio of CAA eluted from the beads in UCP-LF CAA running buffer and the original sample. Each ELISA plate also contained standard curves in the appropriate sample matrices, and CAA-negative controls were run for each condition tested during optimization.

The first parameter optimized was the size of PAMAM conjugated to the magnetic particles. The size of these iteratively synthesized and radially symmetric polymers is defined by generation $(\mathrm{G})$, which is directly related to the number of terminal functionalities $(n)$, where $n=2^{\mathrm{G}+2}$. Thus, optimization of dendrimer size was performed by evaluating CAA capture efficiency of a panel of magnetic particles with PAMAM of varying generations, the concentrations of which were normalized to the number of terminal functionalities during bead functionalization. A constant bead volume $(20 \mu \mathrm{l})$ was incubated with $1 \mathrm{ml}$ of $4 \mathrm{ng} \mathrm{ml}{ }^{-1}$ CAA in PBST with $0.1 \%$ BSA for 5 minutes. As shown in Fig. 1A, the smallest PAMAM size required for maximal CAA capture was G 4.0.

Next, the concentration of PAMAM G 4.0 in the bead conjugation reaction, and therefore the degree of bead surface functionalization, was optimized. Both CAA capture and elution were evaluated. As shown in Fig. 1B, PAMAM G 4.0 maintained optimum capture efficiency at nearly all concentrations of dendrimer. However, recovery of CAA in the elution step was optimal at low PAMAM 4.0 concentrations. Thus, the lowest PAMAM concentration tested, $78 \mu \mathrm{M}$, was chosen as the optimal dendrimer concentration for particle functionalization.

Finally, the total volume of beads required to fully capture and recover CAA from $2 \mathrm{ml}$ of spiked, non-endemic control urine was determined. For this experiment, beads were functionalized with PAMAM G 4.0 at the optimum conditions determined above. Results confirm that $50 \mu \mathrm{l}$ of PAMAM-functionalized magnetic beads achieved full capture and recovery in $2 \mathrm{ml}$ urine samples containing $1 \mathrm{ng} \mathrm{ml}^{-1} \mathrm{CAA}$ (Fig. 1C).

\section{Characterization of PAMAM bead robustness and stability}

The stability and robustness of the PAMAM-functionalized magnetic beads were investigated in order to evaluate their applicability to point-of-care settings and tolerance of sample matrix variation. First, the stability of the PAMAM-functionalized beads in solution (MES pH 5 0.1\% Tween-20) was studied over time for room temperature and $4{ }^{\circ} \mathrm{C}$ storage conditions. The functionalized particles effectively captured all CAA spiked into buffered solutions for the duration of time study (46 days) at both storage temperatures, demonstrating remarkable solution stability, even in ambient conditions (Fig. 2A).

Next, the particle performance in variable matrices was determined, since urine is known to have variable $\mathrm{pH}$ values between 5 and 7 , as well as a wide osmolality range of 38-1400 mOsm $\mathrm{kg}^{-1} \mathrm{H}_{2} \mathrm{O}$. These variables, in particular, would be expected to influence the electrostatic interactions between positively-charged PAMAM and negatively-charged CAA. To determine the tolerance of the PAMAM-functionalized magnetic particles to changes in $\mathrm{pH}$, AWA-TCA was spiked into phosphate buffers $(10 \mathrm{mM})$ with $\mathrm{pH}$ values ranging from 4 to 10. As shown in Fig. 2B, capture was maintained across all $\mathrm{pH}$ values tested. The good performance in basic solutions can be attributed, in part, to the high $\mathrm{p} K_{\mathrm{a}}$ of 9.32 on the terminal amines on PAMAM G 4.0. ${ }^{30}$ Additionally, while the pI of CAA has not been measured, the predicted $\mathrm{p} K_{\mathrm{a}}$ of the $\beta$-D-glucopyranuronic acid portion of the disaccharide repeating unit on the proteoglycan biomarker is 2.78 , which would account for the good performance observed in acidic solutions (ACD/Labs Software V11.02). Finally, the effect of increasing salt concentration on CAA capture was evaluated by adding $\mathrm{NaCl}$ into non-endemic control urine spiked with AWA-TCA. As shown in Fig. 2C, when the salt concentration of urine was increased by $0.5 \mathrm{M}$ or more, the capture efficiency of the PAMAM-functionalized magnetic particles was markedly decreased. However, it should be noted that an increase of $0.5 \mathrm{M} \mathrm{NaCl}$ corresponds to
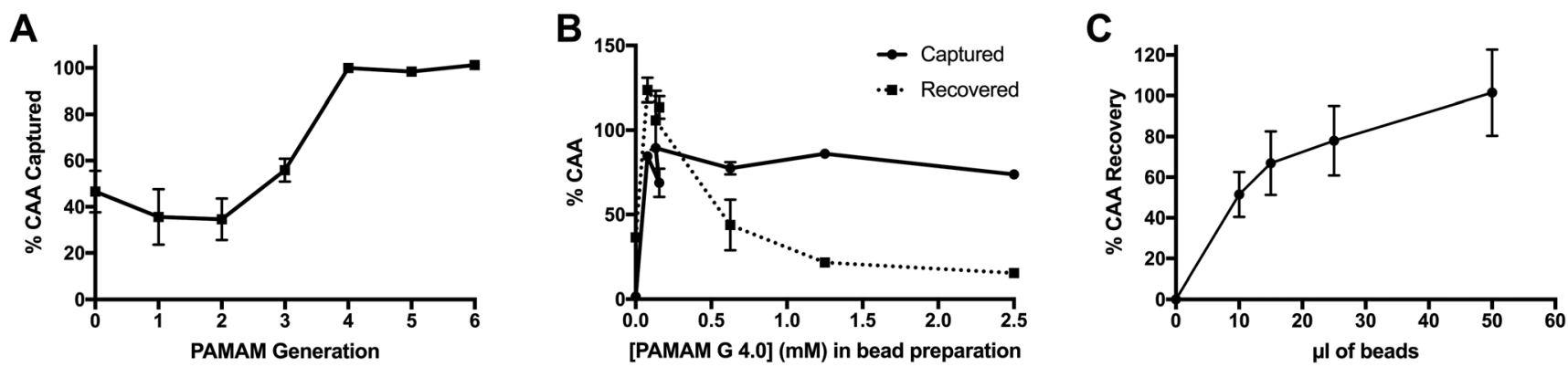

Fig. 1 Optimization of PAMAM-functionalized magnetic particles. (A) Optimal dendrimer size was determined to be PAMAM G 4.0 for full CAA capture. (B) Extent of particle functionalization was found to significantly impact CAA elution, with lower surface densities more readily releasing CAA. (C) In $2 \mathrm{ml}$ spiked urine samples, $50 \mu \mathrm{l}$ of magnetic beads was required to fully recover CAA from solution. 
A

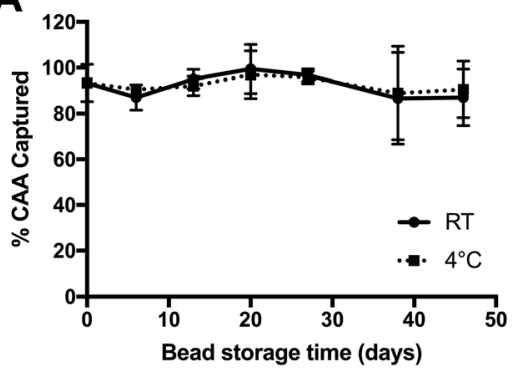

B

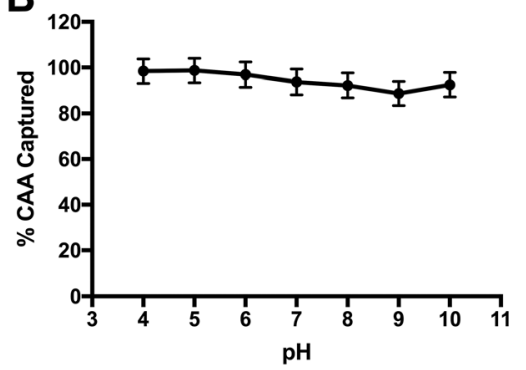

C

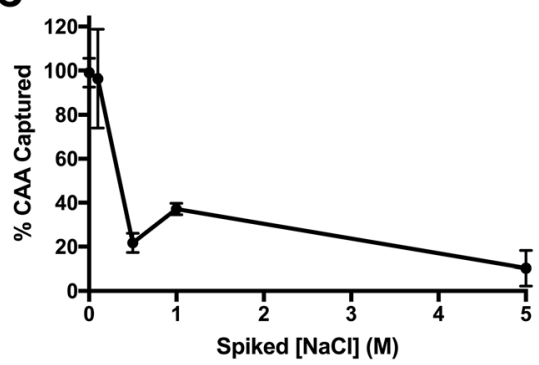

Fig. 2 Evaluation of the stability and robustness of PAMAM-functionalized magnetic particles. (A) After 46 days of storage in solution at RT and $4{ }^{\circ} \mathrm{C}$, the beads maintained full CAA capture efficiency. (B) The PAMAM-based CAA capture was robust to variable $\mathrm{pH}$. (C) High salt concentrations decreased CAA capture efficiency.

an increase in osmolality of 1000 mOsm kg${ }^{-1} \mathrm{H}_{2} \mathrm{O}$. Although the osmolality of the original urine sample is unknown, salt concentrations above the $0.5 \mathrm{M}$ spiked sample would likely be considered abnormal.

\section{Performance of magnetic bead-based CAA concentration protocol}

After optimization and characterization, the PAMAM-functionalized magnetic particles were integrated with the UCP-LF CAA. This workflow required minimal laboratory equipment and could be completed with just 10 minutes of total incubation time. The performance of the bead-based CAA concentration was evaluated against the UCAA10 method in AWA-TCAspiked non-endemic control urine samples. Similar to Corstjens et al., we found the limit of detection of the UCAA10 protocol to be $10 \mathrm{pg} \mathrm{ml}{ }^{-1} \cdot{ }^{21}$ Integration of the optimized PAMAM bead-based CAA concentration protocol resulted in a limit of detection of $0.050 \mathrm{pg} \mathrm{m} \mathrm{m}^{-1}$, achieving the full theoretical 200-fold enhancement expected (Fig. 3).

\section{Patient sample analysis}

Fifteen patient samples from Egypt were analyzed using three sample preparation methods: (1) the original UCAA10 protocol, (2) spin filter-based concentration (UCAA200), and (3) PAMAM magnetic bead-based concentration protocol. Because the

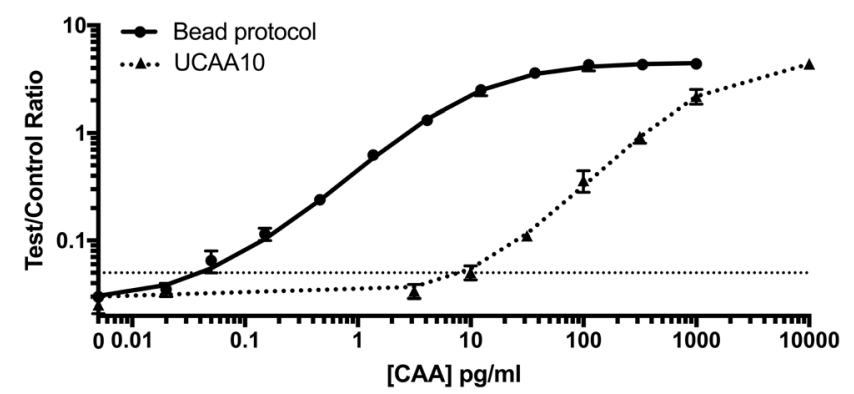

Fig. 3 Performance of magnetic bead-based CAA concentration protocol. Standard curves for the UCAA10 and the PAMAM magnetic bead enhancement method, which successfully concentrated CAA from spiked $2 \mathrm{ml}$ urine samples, achieving full theoretical improvement in LOD of $200 x$. average specific gravity, which is known to correlate well with urine osmolality, of the patient samples $(1.020 \pm 0.007)$ was significantly greater than the non-endemic control urines (1.005) $(P<0.0001)$, patient samples were diluted 10 -fold before the magnetic bead-based concentration protocol was performed. ${ }^{31,32}$ Because of this dilution, the theoretical enhancement possible for the bead-based protocol was 20 -fold. As shown in Fig. 4A, the full theoretical improvement of the LOD was achieved. Additionally, the standard curves for both the bead-based and spin-filter concentration methods aligned as expected, indicating that the two methods are equal in performance despite the large difference in procedure, hands-on time, and laboratory infrastructure required.

Using the PAMAM magnetic bead protocol, UCP-LF CAA signal was enhanced compared to the UCAA10 for all but three
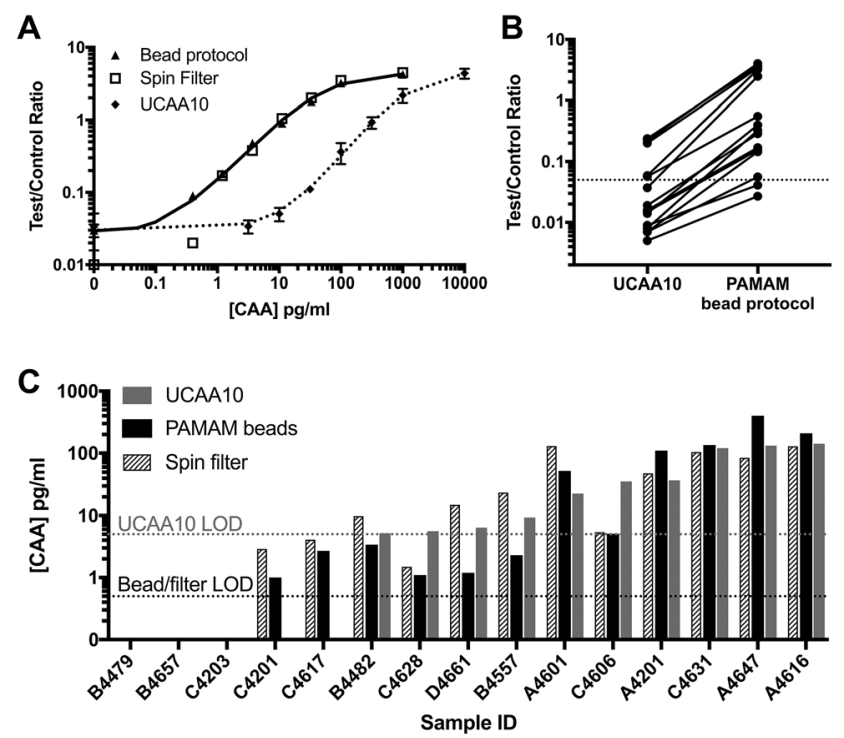

Fig. 4 PAMAM-functionalized magnetic particles successfully concentrated CAA from patient samples. (A) The bead-based protocol performed similarly to spin filter methods in 10x diluted samples. (B) UCP-LF CAA signal was enhanced for all patient samples analyzed with an average enhancement factor of 22x. (C) Results from the bead-based CAA concentration method agreed with the spin filter method developed by Corstjens et al. ${ }^{21}$ 
patient samples tested (Fig. 4B). The average signal enhancement factor was $22 \pm 5$ across all samples, confirming the theoretical concentration limits of the system were reached. The 3 samples that were not enhanced were negative by UCAA10 and remained below the LOD after PAMAM-based concentration. Two UCAA10-negative samples were found to be positive after CAA concentration using the magnetic beads. Additionally, 4 patient samples with CAA concentrations at or near the UCAA10 LOD were found to be definitively positive after employing the magnetic bead concentration protocol. All PAMAM bead-based concentration results were confirmed using spin filters in a manner similar to previous reports, ${ }^{21}$ and good agreement was found between the two methods (Fig. 4C).

\section{Discussion}

Schistosomiasis diagnostic tests capable of detecting lowintensity infections are critical for accurate surveillance and precision mapping of infection prevalence. The UCP-LF CAA is a promising schistosomiasis diagnostic tool that derives its extremely high analytical sensitivity from three components: (1) high-affinity CAA-specific monoclonal antibodies, (2) upconverting phosphor particle labels that effectively eliminate background signal, and (3) a sample preparation step that concentrates CAA from a large volume sample into a smaller volume that can be applied to the test. However, the current protocol for CAA concentration is time-consuming and employs spin filters, requiring laboratory infrastructure that prevents use of the UCP-LF CAA assay at the point of care.

To address this challenge, we have developed and optimized a field-friendly method for CAA concentration from large-volume urine samples. The protocol relies on electrostatic interactions between the negatively-charged CAA biomarker and positively-charged PAMAM dendrimers functionalized to the surface of magnetic particles. These charge-based interactions occur in less than 5 minutes, after which an external magnet is used to remove the CAA-PAMAM-magnetic particle complexes from the large-volume $(2 \mathrm{ml})$ sample and into a small-volume elution buffer. High salt concentration in the elution buffer disrupts the charge-based interactions between CAA and PAMAM, releasing CAA into solution. This concentrated CAA solution is then applied to the UCP-LF CAA test.

During optimization, we observed that increasing PAMAM concentration in bead synthesis beyond the optimum $78 \mu \mathrm{M}$ resulted in decreased CAA recovery, even though CAA capture remained high. This is likely because increasing PAMAM concentration led to a higher density of positive charges on the surface of the beads, resulting in a greater number of interactions between the beads and negatively-charged CAA and making it harder to elute the biomarker. The PAMAMfunctionalized magnetic beads were found to be extremely stable in solution in varied storage conditions, and the protocol tolerated variation in sample $\mathrm{pH}$. When applied to $2 \mathrm{ml}$ CAA-spiked urine samples, the bead-based concentration protocol improved the detection limit of the UCP-LF CAA assay from $10 \mathrm{pg} \mathrm{ml}^{-1}$ to $0.05 \mathrm{pg} \mathrm{ml}^{-1}$, achieving the full theoretical 200-fold enhancement factor and performing just as well as the more resource-intensive spin filter method.

The developed magnetic bead-based assay was then applied to 15 patient samples from Egypt. In order to achieve UCP-LF CAA signal enhancement, we found that the patient samples had to be diluted 10-fold. We hypothesize that high salt concentrations in undiluted endemic patient samples relative to nonendemic control urine disrupted the electrostatic interactions between PAMAM and CAA. Indeed, the average specific gravity, which correlates with urine osmolality, ${ }^{31,32}$ of the endemic patient samples was significantly higher than that of nonendemic control urine. Nonetheless, in 10x-diluted patient samples, the PAMAM-functionalized magnetic particles successfully concentrated CAA, resulting in an average UCP-LF CAA signal enhancement factor of $22 \times$, indicating that this equipment-free protocol reached its full theoretical potential. Additionally, the bead-based concentration method identified 2 positive samples that were otherwise negative according to the unenhanced UCAA10 protocol and provided definitively positive results for 4 samples which had CAA concentrations at or below the detection limits of the UCAA10. These data demonstrate that electrostatic interactions between positively-charged PAMAM dendrimers and the negatively-charged Schistosoma CAA biomarker can be leveraged for equipment-free enhancement of the UCP-LF CAA assay. This robust, magnetic-bead based strategy eliminates the need for laboratory infrastructure for urine sample preparation, bringing the ultrasensitive schistosomiasis detection one step closer to application at the point of care.

Limitations of this study include the relatively small patient sample population tested and the lack of non-CAA-based measurements of disease burden in patient samples. Thus, in the future, the developed protocol must be validated on a larger set of fully-characterized samples in order to fully assess its diagnostic sensitivity and specificity. Although the developed CAA concentration protocol adds to the cost of the UCP-LF CAA assay ( $\sim \$ 3.00 /$ test $)$, the magnetic bead-based biomarker concentration is more affordable than the infrastructure-intensive spin filter concentration step $(\sim \$ 4.00 /$ test $)$. Additionally, while the use of magnetic beads eliminates the need for laboratory equipment for CAA concentration, the protocol requires many user steps. A device that fully integrates sample preparation step the lateral flow assay, similar to those developed by Ricks et al. and Bauer et al., could fill the need to further simplify CAA concentration at the point of care. ${ }^{33,34}$ Finally, PAMAM-functionalization of paper membranes (e.g. cellulose) could open the door to paper fluidic devices that combine the advantages of sample preparation and sensitive detection into a single, affordable, and integrated device applicable in a field setting.

\section{Conclusion}

We have developed a robust and equipment-free sample preparation method for concentration of the CAA biomarker for 
schistosomiasis that leverages electrostatic interactions between positively-charged PAMAM dendrimers and the negatively-charged proteoglycan biomarker. When coupled with the highly sensitive UCP-LF CAA assay, this sample preparation method resulted in a 200-fold improvement in CAA detection, performing just as well as infrastructure-intensive spin filter methods. The developed method demonstrated full theoretical enhancement in patient samples, identifying 2 samples as positive that otherwise would have been negative on an unenhanced test. Thus, the PAMAM-functionalized magnetic bead-based CAA concentration method represents a promising step toward ultrasensitive schistosomiasis detection at the point of care.

\section{Conflicts of interest}

There are no conflicts to declare.

\section{Acknowledgements}

The authors would like to thank T. F. Scherr and N. M. Adams (VU) for designing and printing the custom magnetic rack adaptor, C. J. de Dood (LUMC) for preparing all qualityassured UCP-LF CAA reagents and LF strip materials, and M. F. Richards (VU) for critical comments in the preparation of this manuscript. This work was supported by the Bill and Melinda Gates Foundation (OP1160703), the Vanderbilt Laboratories for Innovation in Global Health Technologies, and Leiden University Medical Center. C. F. M. acknowledges support from the National Science Foundation (NSF) Graduate Research Fellowship (DGE-1445197) and an international travel allowance through the NSF Graduate Research Opportunities Worldwide (GROW).

\section{References}

1 World Health Organization, Schistosomiasis, http://www. who.int/schistosomiasis/en/, (accessed 27 January 2018).

2 World Health Organization, Schistosomiasis (Bilharzia), http://www.afro.who.int/health-topics/schistosomiasis-bilharzia, (accessed 27 January 2018).

3 World Health Organization, Schistosomiasis: progress report 2001-2011, strategic plan 2012-2020, World Health Organization, Geneva, Switzerland, 2013.

4 D. G. Colley, A. L. Bustinduy, W. E. Secor and C. H. King, Human schistosomiasis, Lancet, 2014, 383, 2253-2264.

5 J. Utzinger, S. L. Becker, L. van Lieshout, G. J. van Dam and S. Knopp, New diagnostic tools in schistosomiasis, Clin. Microbiol. Infect., 2015, 21, 529-542.

6 R. A. Braunmunzinger and B. A. Southgate, Repeatability and Reproducibility of Egg Counts of Schistosoma haematobium in Urine, Trop. Med. Parasitol., 1992, 43, 149-154.

7 G. J. van Dam, J. H. Wichers, T. M. F. Ferreira, D. Ghati, A. van Amerongen and A. M. Deelder, Diagnosis of Schistosomiasis by Reagent Strip Test for Detection of
Circulating Cathodic Antigen, J. Clin. Microbiol., 2004, 42, 5458-5461.

8 D. G. Colley, S. Binder, C. Campbell, C. H. King, L.-A. T. Tchuenté, E. K. N'Goran, B. Erko, D. M. S. Karanja, N. B. Kabatereine, L. van Lieshout and S. Rathbun, A FiveCountry Evaluation of a Point-of-Care Circulating Cathodic Antigen Urine Assay for the Prevalence of Schistosoma mansoni, Am. J. Trop. Med. Hyg., 2013, 88, 426-432.

9 M. Adriko, C. J. Standley, B. Tinkitina, E. M. Tukahebwa, A. Fenwick, F. M. Fleming, J. C. Sousa-Figueiredo, J. R. Stothard and N. B. Kabatereine, Evaluation of circulating cathodic antigen (CCA) urine-cassette assay as a survey tool for Schistosoma mansoni in different transmission settings within Bugiri District, Uganda, Acta Trop., 2014, 136, 50-57.

10 E. A. Ochodo, G. Gopalakrishna, B. Spek, J. B. Reitsma, L. van Lieshout, K. Polman, P. Lamberton, P. M. Bossuyt and M. M. Leeflang, Circulating antigen tests and urine reagent strips for diagnosis of active schistosomiasis in endemic areas, Cochrane Database Syst. Rev., 2015, 3, CD009579.

11 A. M. S. Silveira, E. G. D. Costa, D. Ray, B. M. Suzuki, M. H. Hsieh, L. A. O. de Fraga and C. R. Caffrey, Evaluation of the CCA Immuno-Chromatographic Test to Diagnose Schistosoma mansoni in Minas Gerais State, Brazil, PLoS Negl. Trop. Dis., 2016, 10, e0004357.

12 H. Greter, S. J. Krauth, B. N. R. Ngandolo, I. O. Alfaroukh, J. Zinsstag and J. Utzinger, Validation of a Point-of-Care Circulating Cathodic Antigen Urine Cassette Test for Schistosoma mansoni Diagnosis in the Sahel, and Potential Cross-Reaction in Pregnancy, Am. J. Trop. Med. Hyg., 2016, 94, 361-364.

13 M. Casacuberta, S. Kinunghi, B. J. Vennervald and A. Olsen, Evaluation and optimization of the Circulating Cathodic Antigen (POC-CCA) cassette test for detecting Schistosoma mansoni infection by using image analysis in school children in Mwanza Region, Tanzania, Parasite Epidemiol. Control, 2016, 1, 105-115.

14 N. Kittur, J. D. Castleman, C. H. C. Jr, C. H. King and D. G. Colley, Comparison of Schistosoma mansoni Prevalence and Intensity of Infection, as Determined by the Circulating Cathodic Antigen Urine Assay or by the KatoKatz Fecal Assay: A Systematic Review, Am. J. Trop. Med. Hyg., 2016, 94, 605-610.

15 A. Danso-Appiah, J. Minton, D. Boamah, J. Otchere, R. H. Asmah, M. Rodgers, K. M. Bosompem, P. Eusebi and S. J. De Vlas, Accuracy of point-of-care testing for circulatory cathodic antigen in the detection of schistosome infection: systematic review and meta-analysis, Bull World Health Organ, 2016, 94, 522-533A.

16 J. T. Coulibaly, S. Knopp, N. A. N’Guessan, K. D. Silué, T. Fürst, L. K. Lohourignon, J. K. Brou, Y. K. N'Gbesso, P. Vounatsou, E. K. N'Goran and J. Utzinger, Accuracy of Urine Circulating Cathodic Antigen (CCA) Test for Schistosoma mansoni Diagnosis in Different Settings of Côte d'Ivoire, PLoS Negl. Trop. Dis., 2011, 5, e1384. 
17 P. L. A. M. Corstjens, L. van Lieshout, M. Zuiderwijk, D. Kornelis, H. J. Tanke, A. M. Deelder and G. J. van Dam, Up-Converting Phosphor Technology-Based Lateral Flow Assay for Detection of Schistosoma Circulating Anodic Antigen in Serum, J. Clin. Microbiol., 2008, 46, 171-176.

18 P. L. A. M. Corstjens, C. J. de Dood, D. Kornelis, E. M. T. K. Fat, R. A. Wilson, T. M. Kariuki, R. K. Nyakundi, P. T. Loverde, W. R. Abrams, H. J. Tanke, L. van Lieshout, A. M. Deelder and G. J. van Dam, Tools for diagnosis, monitoring and screening of Schistosoma infections utilizing lateral-flow based assays and upconverting phosphor labels, Parasitology, 2014, 141, 1841-1855.

19 A. Agnew, A. J. C. Fulford, N. D. Jonge, F. W. Krijger, M. Rodriguez-Chacon, V. Gutsmann and A. M. Deelder, The relationship between worm burden and levels of a circulating antigen (CAA) of five species of Schistosoma in mice, Parasitology, 1995, 111, 67-76.

20 P. L. A. M. Corstjens, S. Li, M. Zuiderwijk, K. Kardos, W. R. Abrams, R. S. Niedbala and H. J. Tanke, Infrared upconverting phosphors for bioassays, IEE Proc.: Nanobiotechnol., 2005, 152, 64-72.

21 P. L. Corstjens, R. K. Nyakundi, C. J. de Dood, T. M. Kariuki, E. A. Ochola, D. M. Karanja, P. N. Mwinzi and G. J. van Dam, Improved sensitivity of the urine CAA lateral-flow assay for diagnosing active Schistosoma infections by using larger sample volumes, Parasites Vecto, 2015, 8, 241.

22 G. J. van Dam, J. Xu, R. Bergquist, C. J. de Dood, J. Utzinger, Z.-Q. Qin, W. Guan, T. Feng, X.-L. Yu, J. Zhou, M. Zheng, X.-N. Zhou and P. L. A. M. Corstjens, An ultrasensitive assay targeting the circulating anodic antigen for the diagnosis of Schistosoma japonicum in a low-endemic area, People's Republic of China, Acta Trop., 2015, 141, 190-197.

23 S. Knopp, P. L. A. M. Corstjens, A. Koukounari, C. I. Cercamondi, S. M. Ame, S. M. Ali, C. J. de Dood, K. A. Mohammed, J. Utzinger, D. Rollinson and G. J. van Dam, Sensitivity and Specificity of a Urine Circulating Anodic Antigen Test for the Diagnosis of Schistosoma haematobium in Low Endemic Settings, PLoS Negl. Trop. Dis., 2015, 9, e0003752.

24 Y. Vonghachack, S. Sayasone, V. Khieu, R. Bergquist, G. J. van Dam, P. T. Hoekstra, P. L. A. M. Corstjens, B. Nickel, H. Marti, J. Utzinger, S. Muth and P. Odermatt, Comparison of novel and standard diagnostic tools for the detection of Schistosoma mekongi infection in Lao People's Democratic Republic and Cambodia, Infect. Dis. Poverty, 2017, 6, 127.
25 P. L. A. M. Corstjens, P. T. Hoekstra, C. J. de Dood and G. J. van Dam, Utilizing the ultrasensitive Schistosoma upconverting phosphor lateral flow circulating anodic antigen (UCP-LF CAA) assay for sample pooling-strategies, Infect. Dis. Poverty, 2017, 6, 155.

26 A. M. Deelder, G. J. van Dam, D. Kornelis, Y. E. Fillié and R. J. van Zeyl, Schistosoma: analysis of monoclonal antibodies reactive with the circulating antigens CAA and CCA, Parasitology, 1996, 112(Pt 1), 21-35.

27 A. A. Bergwerff, G. J. van Dam, J. P. Rotmans, A. M. Deelder, J. P. Kamerling and J. F. Vliegenthart, The immunologically reactive part of immunopurified circulating anodic antigen from Schistosoma mansoni is a threonine-linked polysaccharide consisting of $\rightarrow$ 6)-(betaD-GlcpA-(1 -> 3))-beta-D-GalpNAc-(1 -> repeating units, J. Biol. Chem., 1994, 269, 31510-31517.

28 A. M. Deelder, N. D. Jonge, O. C. Boerman, Y. E. Fillié, G. W. Hilberath, J. P. Rotmans, M. J. Gerritse and D. W. O. L. Schut, Sensitive Determination of Circulating Anodic Antigen in Schistosoma mansoni Infected Individuals by an Enzyme-Linked Immunosorbent Assay using Monoclonal Antibodies, Am. J. Trop. Med. Hyg., 1989, 40, 268-272.

29 L. V. Lieshout, N. D. Jonge, N. A. E. Masry, M. M. Mansour, F. W. Krijger and A. M. Deelder, Improved Diagnostic Performance of the Circulating Antigen Assay in Human Schistosomiasis by Parallel Testing for Circulating Anodic and Cathodic Antigens in Serum and Urine, Am. J. Trop. Med. Hyg., 1992, 47, 463-469.

30 Y. Niu, L. Sun and R. M. Crooks, Determination of the Intrinsic Proton Binding Constants for Poly(amidoamine) Dendrimers via Potentiometric $\mathrm{pH}$ Titration, Macromolecules, 2003, 36, 5725-5731.

31 S. Leech and M. D. Penney, Correlation of specific gravity and osmolality of urine in neonates and adults., Arch. Dis. Child., 1987, 62, 671-673.

32 G. C. Voinescu, M. Shoemaker, H. Moore, R. Khanna and K. D. Nolph, The Relationship between Urine Osmolality and Specific Gravity, Am. J. Med. Sci., 2002, 323, 39-42.

33 K. M. Ricks, N. M. Adams, T. F. Scherr, F. R. Haselton and D. W. Wright, Direct transfer of HRPII-magnetic bead complexes to malaria rapid diagnostic tests significantly improves test sensitivity, Malar. J., 2016, 15, 399.

34 W. S. Bauer, D. W. Kimmel, N. M. Adams, L. E. Gibson, T. F. Scherr, K. A. Richardson, J. A. Conrad, H. K. Matakala, F. R. Haselton and D. W. Wright, Magnetically-enabled biomarker extraction and delivery system: towards integrated ASSURED diagnostic tools, Analyst, 2017, 142, 1569-1580. 